\title{
Overexpression of miR-15b-5p promotes gastric cancer metastasis by regulating PAQR3
}

\author{
CHENGCHENG ZHAO $^{1}$, YAN LI ${ }^{1,2}$, GANG CHEN $^{3}$, FEN WANG $^{3}$, \\ ZHILI SHEN $^{3}$ and RONGPING ZHOU ${ }^{3}$ \\ ${ }^{1}$ Central Laboratory and ${ }^{2}$ Clinical Laboratory, and ${ }^{3}$ Department of Oncology, \\ The Affiliated Jiangning Hospital of Nanjing Medical University, Nanjing, Jiangsu 210000, P.R. China
}

Received October 12, 2016; Accepted November 23, 2016

DOI: $10.3892 / o r .2017 .5673$

\begin{abstract}
Gastric cancer (GC) is the fifth most common cancer in the world, with 952,000 new cases diagnosed in 2012. Tumor metastasis is the major cause of cancer recurrence and death. miR-15b-5p has been reported to be dysregulated in numerous types of cancers. However, the role of miR-15b-5p in GC metastasis remains unclear. An miRNA microarray was adopted to analyze the miRNA expression profile. By employing quantitative real-time polymerase chain reaction (qRT-PCR), miR-15b-5p expression levels were detected in GC cell lines, tissues and plasma samples. In addition, the effects of miR-15b-5p on cell proliferation, migration and invasion were studied by applying gain-of-function approaches. Moreover, the target of miR-15b-5p was assessed by dual-luciferase assay, and the mechanism underlying the regulation of GC metastasis by miR-15b-5p was assessed by rescue experiments. The results revealed that miR-15b-5p was upregulated in GC cell lines, tissues and plasma samples. A high plasma level of miR-15b-5p was correlated with distant tumor metastasis. In addition, overexpression of miR-15b-5p in GC cells promoted cell proliferation, migration, invasion and epithelial-mesenchymal transition (EMT). Moreover, progestin and adipoQ receptor family member 3 (PAQR3) was found to be a direct target of miR-15b-5p and re-expression of PAQR3 in miR-15b-5p-overexpressing GC cells partly attenuated the proliferation, migration and invasion. These findings revealed that miR-15b-5p promotes the metastasis of GC cells through PAQR3 and may represent a potential biomarker of GC.
\end{abstract}

Correspondence to: Dr Zhili Shen or Dr Rongping Zhou, Department of Oncology, The Affiliated Jiangning Hospital of Nanjing Medical University, 168 Gushan Road, Nanjing, Jiangsu 210000, P.R. China

E-mail: jnyycl@sina.com

E-mail: pinger_523@126.com

Key words: miR-15b-5p, gastric cancer, PAQR3, metastasis

\section{Introduction}

In China, gastric cancer (GC) is the second most common malignancy (1). In 2012, 405,000 new cases were estimated to occur worldwide, which accounted for $42.5 \%$ of the total cases (2). Due to a lack of early detection and efficient screening in China, the majority of patients are often diagnosed with distant metastases, leading to a poor 5-year survival rate $(3,4)$. Therefore, investigation of the molecular pathogenesis of GC and the development of effective diagnostic biomarkers are critical for GC patients.

miRNAs are small non-coding RNAs which play significant roles in the initiation and progression of various types of cancer (5-7). Thus, investigation of the mechanisms underlying the regulation of GC by miRNAs is important. miR-15b belongs to the miR-15 family, which is located on chromosome 3 (8). miR-15b-5p is a mature miRNA spliced from the 5'-end of pre-miR-15b. Aberrant miR-15b expression has been reported in a large number of cancers, and contributes to increased proliferation, reduced apoptosis, tumor metastasis, recurrence and poor patient prognosis (9-12). However, to the best of our knowledge, little has been reported regarding the role of miR-15b-5p in GC.

The present study revealed the expression profile of miRNAs in GC tissues and miR-15b-5p was chosen for further study. The expression pattern of miR-15b-5p was detected in GC cell lines, tissues and plasma samples. Moreover, progestin and adipoQ receptor family member 3 (PAQR3) was found to be a direct target of miR-15b-5p. Furthermore, the mechanism of miR-15b-5p in regulating GC metastasis was analyzed.

\section{Materials and methods}

GC tissues and cell lines. Forty pairs of GC and adjacent nontumor tissues, $100 \mathrm{GC}$ patient blood samples and 100 blood samples from healthy subjects were obtained from the Affiliated Jiangning Hospital of Nanjing Medical University (Nanjing, China). There were no significant differences in sex, age and accompanying diseases between the groups. All the tissue specimens were confirmed by two pathologists and frozen in liquid nitrogen until RNA extraction. The blood samples from the healthy subjects were collected from individuals who were undergoing regular physical check-ups at 
our hospital. The present study was approved by the Ethics Committee of Nanjing Jiangning Hospital and consent forms were obtained from all of the patients.

Four GC cell lines (AGS, BGC-823, SGC-7901 and MGC-803), a GES-1 immortalized human gastric epithelial mucosa cell line and the HeLa cell line were purchased from the Cell Bank of the Chinese Academy of Sciences (Shanghai, China). Cells were grown in RPMI-1640 medium (Invitrogen Life Technologies, Carlsbad, CA, USA), supplemented with $10 \%$ fetal bovine serum (FBS) and $100 \mathrm{U} / \mathrm{ml}$ penicillin/ streptomycin (both from Invitrogen, Carlsbad, CA, USA) at $37^{\circ} \mathrm{C}$ with $5 \% \mathrm{CO}_{2}$ in an incubator (Thermo Fisher Scientific, Waltham, MA, USA).

miRNA expression profiling analysis. Three pairs of GC and adjacent non-tumor tissues were adopted to analyze the miRNA expression profile by miRNA microarray at KangChen Bio-tech (Shanghai, China). The miRNA microarray analysis was performed as previously described (13).

Plasma preparation. Peripheral venous blood $(2 \mathrm{ml})$ from patients or healthy individuals was collected in a tube containing EDTA and centrifuged at $1,800 \mathrm{x} \mathrm{g}$ for $5 \mathrm{~min}$ to isolate the plasma. Then, the plasma was stored at $-80^{\circ} \mathrm{C}$ until RNA extraction.

$q R T-P C R$. TRIzol and TRIzol LS reagents (both from Invitrogen) were respectively used to extract RNA from tissues and cells and that from plasma. The RNA concentration was quantitated on a protein nucleic acid spectrophotometer (BioDrop, Cambridge, UK). Complementary DNA was obtained using a reverse-transcription PrimeScript ${ }^{\mathrm{TM}}$ RT reagent kit with gDNA Eraser (Takara Bio, Inc., Otsu, Japan) according to the manufacturer's instructions. The qRT-PCR reaction was performed on an ABI StepOne Plus System (Applied Biosystems; Thermo Fisher Scientific, Inc.). The relative expression of miR-15b-5p was calculated using the $2^{-\Delta \Delta \mathrm{Ct}}$ method (14), and U6 served as the internal control. qRT-PCR primer set for miR-15b-5p (miRQ0000417-1-1) and U6 (MQP-0202) were purchased from RiboBio (Guangzhou, China). The primer sequences were as follows: PAQR3 forward, 5'-CTCAAGGACAACCCGTACATCAC-3' and reverse, 5'-AAACTTTTGATACACAGCCTGGAC-3'; RECK forward, 5'-TGGCAAGAGTTTGATCGCTT-3' and reverse, 5'-ATGGCTCCTTGATCTGACTGTG-3'; SMAD7 forward, 5'-ATGCTGTGCCTTCCTCCGCTG-3' and reverse, 5'-CCAC GCACCAGTGTGACCGA-3'; $\beta$-actin forward, 5'-GATCATT GCTCCTCCTGAGC-3' and reverse, 5'-ACTCCTGCTTGCT GATCCAC-3'.

Cell transfection. BGC-823 and SGC-7901 cell lines were used for cell transfection. Cells $\left(3 \times 10^{5}\right)$ were seeded into a 6-well culture plate (Corning, Corning, Inc., Corning, NY, USA) and transiently transfected with miRNA using Lipofectamine $^{\circledR} 2000$ (Invitrogen) after reaching 70-90\% confluency. At $6 \mathrm{~h}$ after transfection, the medium was replaced with fresh medium. A final concentration of $50 \mathrm{nM}$ miR-15b-5p mimic and negative control (RiboBio), $2.5 \mathrm{ng}$ of PAQR3 expression vector and control vector (SunShineBio, Nanjing, China) were used for transfection.
Cell proliferation assay. Cell proliferation ability was measured by Cell Counting Kit-8 (CCK-8) assay (Dojindo Molecular Technologies, Kumamoto, Japan). Cells $\left(3 \times 10^{3}\right)$ were planted into a 96-well plate (Corning) and transfected as described above. At 24, 48 and $72 \mathrm{~h}$ after transfection, the medium was replaced with a mixture of $100 \mu \mathrm{l}$ of fresh medium and $10 \mu \mathrm{l}$ of CCK-8. After a 2-h incubation, the optical density (OD) value was measured on a microplate reader (iMark; Bio-Rad Laboratories, Inc., Hercules, CA, USA) at $450 \mathrm{~nm}$.

Wound healing assay. Cells were transfected as described above, and starved for $12 \mathrm{~h}$ before scratching. Pipette tips $(20 \mu \mathrm{l})$ were used to generate a wound. At 0 and $72 \mathrm{~h}$ after the scratch, images of the wound areas were captured under an inverted microscope (IX51; Olympus Co., Tokyo, Japan) at a magnification of $\mathrm{x} 200$.

Transwell assay. Twenty-four hours after transfection, the cells were starved for $8 \mathrm{~h}$, trypsinized (Gibco, Grand Island, NY, USA) and suspended in serum-free medium at a concentration of 3x10 $/ \mathrm{ml}$. Complete RPMI-1640 medium (600 $\mu \mathrm{l})$ was added into the lower well and $100 \mu \mathrm{l}$ of cell suspension was added into the upper chamber which was coated with Matrigel (BD Biosciences, San Jose, CA, USA). After incubating for $48 \mathrm{~h}$, the upper chamber (Corning) was wiped with a swab to remove cells on the upper surface, and then stained with crystal violet (Beyotime Institute of Biotechnology, Shanghai, China) for $20 \mathrm{~min}$. After washing with distilled water, the cells that had invaded to the lower surface were counted and captured under a microscope (Olympus) at a magnification of $\mathrm{x} 200$. Five random and different vision fields were selected to count the invasive cell number.

Western blotting. Cells were lysed using radioimmunoprecipitation assay (RIPA) lysis buffer (Beyotime Institute of Biotechnology) on ice for $5 \mathrm{~min}$, and were then centrifugalized at $12,000 \mathrm{x} \mathrm{g}$ for $5 \mathrm{~min}$ at $4^{\circ} \mathrm{C}$ to extract the protein. The concentration of the protein was determined by the BCA method (BCA protein assay kit; Beyotime Institute of Biotechnology). Proteins (30 $\mu \mathrm{g})$ were separated on $10 \%$ SDS-PAGE and transferred to a polyvinylidene fluoride (PVDF) membrane (EMD Millipore, Billerica, MA, USA). The membranes were blocked with $5 \%$ non-fat milk for $1 \mathrm{~h}$, and then incubated in the primary antibodies at $4^{\circ} \mathrm{C}$ overnight, including $\beta$-actin (A5441, mouse monoclonal, 1:8,000; Sigma-Aldrich, St. Louis, MO, USA), E-cadherin (ab76055, mouse monoclonal; 1:500; Abcam, Cambridge, UK), vimentin (sc-6260, mouse monoclonal, 1:500), PAQR3 (sc-161992, goat polyclonal, 1:500) (both from Santa Cruz Biotechnology, Santa Cruz, CA, USA). After being washed in Tris-buffered solution with Tween-20 (TBST), the membranes were incubated in the secondary antibody (ZB-2306, rabbit anti-goat and ZB-2305, goat anti-mouse 1:10,000; ZSGB-Bio, Ltd., Beijing, China) for $2 \mathrm{~h}$. The protein bands were detected using an enhanced chemiluminescence (ECL) kit (Pierce, Rockford, IL, USA) on a FluorChem E System (ProteinSimple, San Jose, CA, USA).

Dual-luciferase assay. The wild-type (WT) and mutant (Mut) binding sequences of miR-15b-5p on the 3 ' untranslated region 


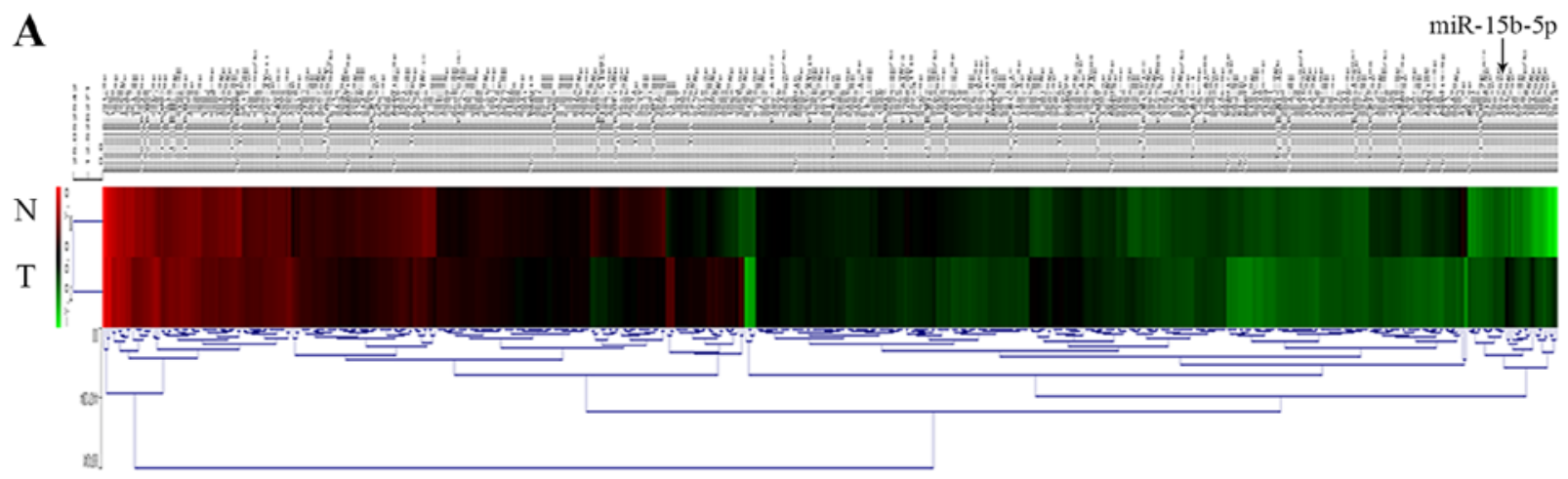

B

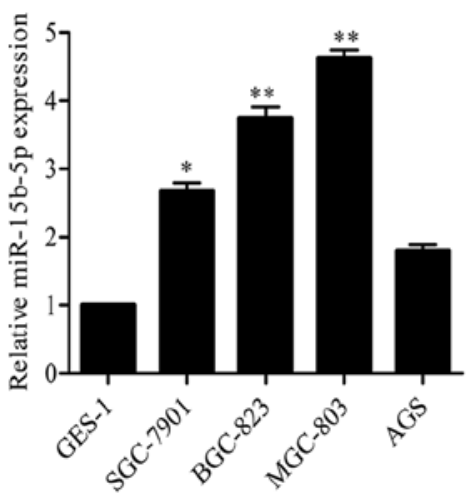

C

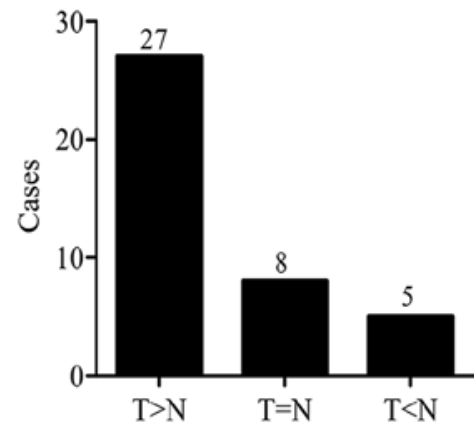

D

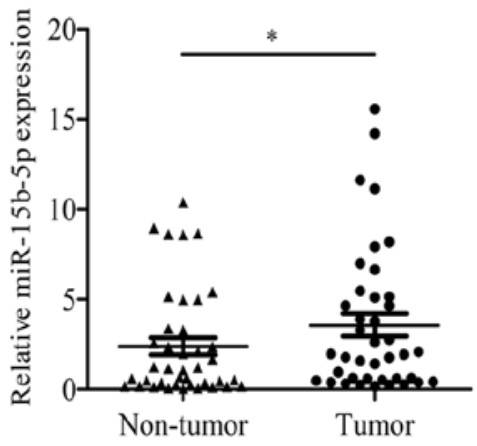

Figure 1. miR-15b-5p is upregulated in GC cell lines and tissues. (A) Microarray analysis of GC tissues. (B) The expression level of miR-15b-5p in four GC cell lines and normal gastric epithelium GES-1 cells. (C) Cases of upregulated miR-15b-5p in GC tissues. T, tumor; N, non-tumor. (D) Scatter plots of miR-15b-5p fold-change in GC and matched non-tumor tissues; ${ }^{*} \mathrm{P}<0.05,{ }^{* *} \mathrm{P}<0.01$.

(3'UTR) of PAQR3 were synthesized (SunShineBio) and cloned into the pMIR-reportor vector (Invitrogen), which were respectively named pMIR-report-PAQR3-3'UTR-WT and pMIR-report-PAQR3-3'UTR-Mut. HeLa cells $\left(1 \times 10^{5}\right)$ were seeded into a 24-well plate (Corning) and cultured overnight untilreaching 70-90\% confluency.pMIR-report $\beta$-galactosidase reporter plasmid (500 ng), pMIR-report-PAQR3-3'UTR-WT (500 ng) or pMIR-report-PAQR3-3'UTR-Mut (500 ng), miR-15b-5p mimic $(50 \mathrm{nM})$ or negative control $(50 \mathrm{nM})$ were co-transfected using Lipofectamine 2000. Cells were collected at $48 \mathrm{~h}$ post-transfection, and luciferase activities were measured using the Dual-Luciferase Reporter Assay System (Promega, Madison, WI, USA) on a TD-20/20 Luminometer (Turner BioSystems, Sunnyvale, CA, USA) according to the manufacturer's instructions.

Statistical analysis. The data are expressed as the mean \pm SD from at least three independent experiments. Student's t-test (SPSS Statistics 17; SPSS, Inc., Chicago, IL, USA) was adopted to determine the statistical significance between the groups. A P-value of $<0.05$ was considered to indicate a statistically significant result.

\section{Results}

miRNA expression profile in GC tissues. Three GC and paired non-tumor tissues were respectively pooled, and subjected to microarray analysis. The heat map is shown in Fig. 1A.
Eighty-two miRNAs were upregulated (ratio of tumor to non-tumor tissue $\geq 1.5$ ), while 179 were significantly downregulated (ratio of tumor to non-tumor tissue $<1.5$ ), among which, miR-15b-5p was upregulated 7.6-fold and selected for further research.

miR-15b-5p is upregulated in GC cell lines and tissues. In order to verify the miRNA array results, miR-15b-5p expression was first detected in GC cell lines. The results showed that miR-15b-5p expression levels were higher in $4 \mathrm{GC}$ cell lines (SGC-7901, BGC-823, MGC-803 and AGS) than that in the normal gastric epithelium GES-1 cells (Fig. 1B). In 40 pairs of tumor and matched non-tumor tissue, miR-15b-5p was upregulated in $72.5 \%$ (27/40) of the cases (Fig. 1C). miR-15b-5p expression levels were much higher in the tumor tissues than that in the non-tumor tissues (Fig. 1D). In addition, the expression pattern of miR-15b-5p and clinicopathological characteristics of the GC patients were compared, and the data showed that miR-15b-5p expression was correlated with the degree of tumor invasion and lymph node metastasis (Table I).

Plasma miR-15b-5p level is correlated with distant metastasis of $G C$. To evaluate the diagnostic value of plasma miR-15b-5p in GC metastasis, the expression levels of plasma miR-15b-5p were first examined in $100 \mathrm{GC}$ patients and 100 cancer-free healthy control subjects. The results showed that miR-15b-5p expression levels in the GC group were higher than those in the control group $(\mathrm{p}=0.011$; Fig. $2 \mathrm{~A})$. Next, GC plasma 
Table I. Relationship between miR-15b-5p expression and clinicopathological characteristics of the GC patients.

\begin{tabular}{|c|c|c|c|c|}
\hline \multirow[b]{2}{*}{$\begin{array}{l}\text { Patient } \\
\text { characteristics }\end{array}$} & \multicolumn{3}{|c|}{$\begin{array}{l}\text { Relative miR-15b-5p } \\
\text { expression }\end{array}$} & \multirow[b]{2}{*}{ P-value } \\
\hline & $\begin{array}{c}\text { Low } \\
(n=13)\end{array}$ & $\begin{array}{l}\text { High } \\
(n=27)\end{array}$ & $\begin{array}{l}\text { Total } \\
(\mathrm{n}=40)\end{array}$ & \\
\hline Sex & & & & 0.890 \\
\hline Male & 8 & 16 & 24 & \\
\hline Female & 5 & 11 & 16 & \\
\hline Age (years) & & & & 0.768 \\
\hline$\leq 60$ & 3 & 9 & 12 & \\
\hline$>60$ & 10 & 18 & 28 & \\
\hline Lauren's classification & & & & 0.305 \\
\hline Diffuse type & 7 & 19 & 26 & \\
\hline Intestinal type & 6 & 8 & 14 & \\
\hline Differentiation & & & & 0.720 \\
\hline Poor & 8 & 15 & 23 & \\
\hline Moderate/well & 5 & 12 & 17 & \\
\hline Degree of invasion & & & & $0.029^{\mathrm{a}}$ \\
\hline Tunica mucosa & 8 & 7 & 15 & \\
\hline Mucous layer outside & 5 & 20 & 25 & \\
\hline Lymph node metastasis & & & & $0.042^{\mathrm{a}}$ \\
\hline Yes & 4 & 19 & 23 & \\
\hline No & 9 & 8 & 17 & \\
\hline
\end{tabular}

${ }^{\mathrm{a} P}<0.05$. GC, gastric cancer.

specimens were categorized into a distant metastasis group $(n=40)$ and a non-metastasis group $(n=60)$ according to the diagnosis. The data indicated that the distant metastasis group had a higher miR-15b-5p level than that of the non-metastasis group ( $\mathrm{p}=0.018$; Fig. $2 \mathrm{~B})$.

miR-15b-5p promotes GC metastasis. miR-15b-5p mimics or negative control were introduced into BGC-823 and SGC-7901 cells to observe the biological function of miR-15b-5p in GC cells. After transient transfection, enforced expression levels of miR-15b-5p were detected in the two GC cell lines (Fig. 3A). The effect of miR-15b-5p on gastric cell proliferation was evaluated by CCK-8 assay. Data showed that when compared with the control cells, exogenous expression of miR-15b-5p in the BGC-823 and SGC-7901 cells caused significant increases in cell growth rates (Fig. 3B). To determine the influence of miR-15b-5p on cell migration, a wound healing assay was employed. The results indicated that miR-15b-5p overexpression promoted the migratory ability of both GC cell lines (Fig. 3C). The invasive capacity of miR-15b-5p was detected by Transwell assay. Enhanced miR-15b-5p expression increased the number of cells that invaded to the lower surface of the membrane (Fig. 3D). The levels of epithelial marker E-cadherin and mesenchymal marker vimentin were further examined to confirm whether miR-15b-5p participates in the epithelial-mesenchymal transition (EMT) process. The
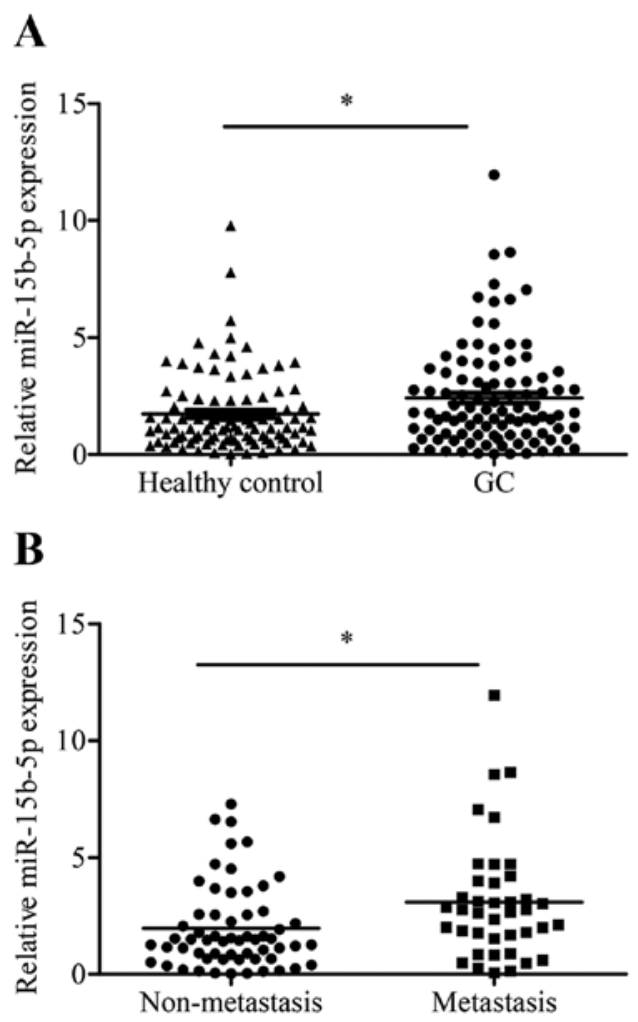

Figure 2. miR-15b-5p is upregulated in GC patient plasma. (A) The relative expression difference of miR-15b-5p in plasma of healthy control subjects and GC patients. (B) The relative expression difference of miR-15b-5p in the non-metastasis group and the metastasis group of GC patients; ${ }^{*} \mathrm{P}<0.05$. GC, gastric cancer.

results showed that, when compared with the control cells, the expression of E-cadherin was decreased while the expression of vimentin was increased in the miR-15b-5p-overexpressing GC cells (Fig. 3E).

PAQR3 is a target of miR-15b-5p in GC. Online software programs (TargetScan and miRDB) were used to predict the target of miR-15b-5p, and numerous genes were found to contain the binding sequence of miR-15b-5p. Among these candidates, PAQR3, RECK and SMAD7 were identified, as their functions were related to cancer metastasis. The qRT-PCR results showed that miR-15b-5p overexpression did not have marked effects on RECK and SMAD7 expression, while PAQR3 was significantly decreased in the miR-15b-5poverexpressing BGC-823 and SGC-7901 cells (Fig. 4A and B). Luciferase reporter assay was performed to confirm the result. Wild-type and mutant binding sequences of miR15b-5p on 3'UTR of PAQR3 were amplified (Fig. 4C), and the results showed that miR-15b-5p decreased the luciferase activity of pMIR-report-PAQR3-3'UTR-WT but not of pMIRreport-PAQR3-3'UTR-Mut (Fig. 4D). Collectively, these data indicated that PAQR3 is a direct target of miR-15b-5p.

Overexpression of PAQR3 partially rescues miR-15b-5pinduced cell proliferation, migration and invasion. Rescue experiments were performed to verify whether miR-15b-5p regulates GC metastasis by targeting PAQR3. PAQR3 expression vector and control vector were transfected into the 
A

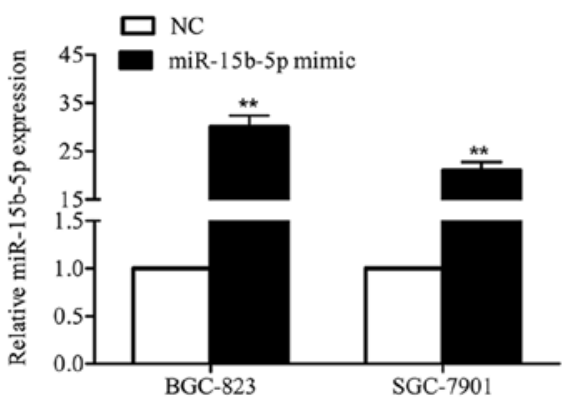

C

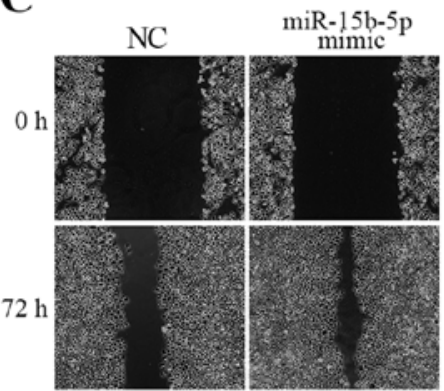

BGC- 823

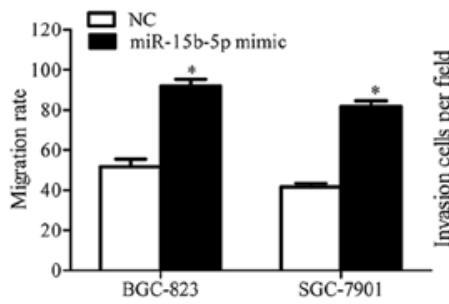

B

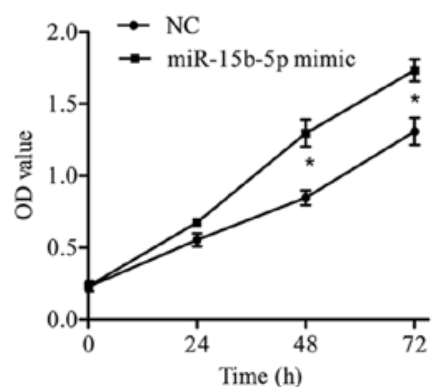

D
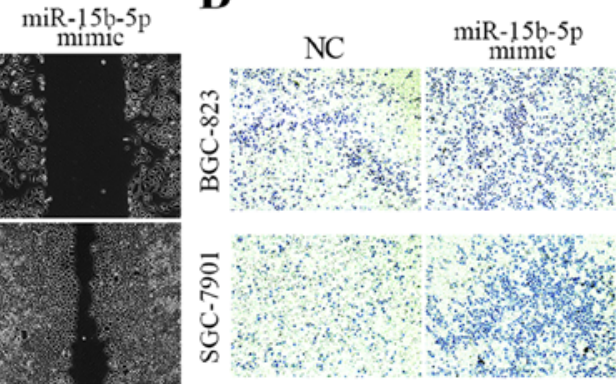

SGC-7901

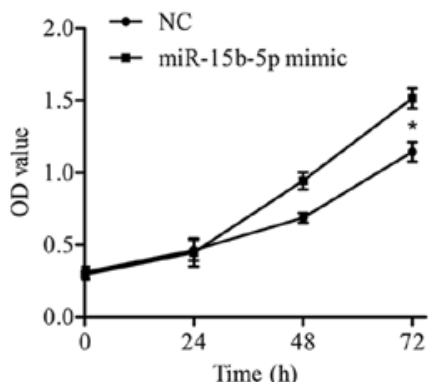

$\mathbf{E}$

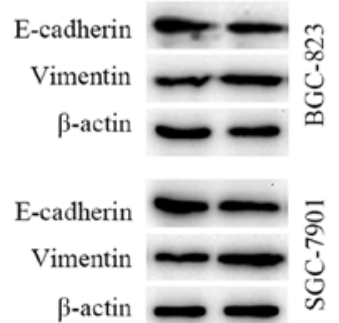

BGC- 823
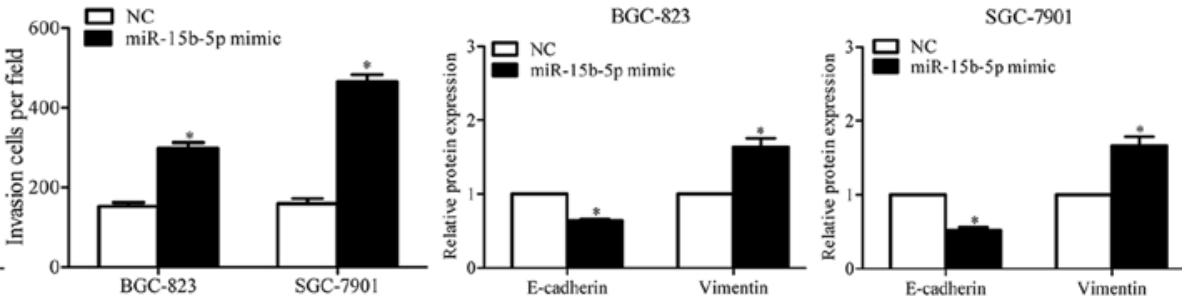

Figure 3. Overexpression of miR-15b-5p promotes GC cell metastasis. (A) The expression level of miR-15b-5p was significantly increased after transfection of miR-15b-5p mimics in the BGC-823 and SGC-7901 cell lines. Overexpression of miR-15b-5p promoted (B) GC cell proliferation, (C) migration and (D) invasion. (E) Overexpression of miR-15b-5p downregulated E-cadherin and upregulated vimentin in both GC cell lines; ${ }^{*} \mathrm{P}<0.05$, ${ }^{* *} \mathrm{P}<0.01$ vs. NC. NC, negative control.

A

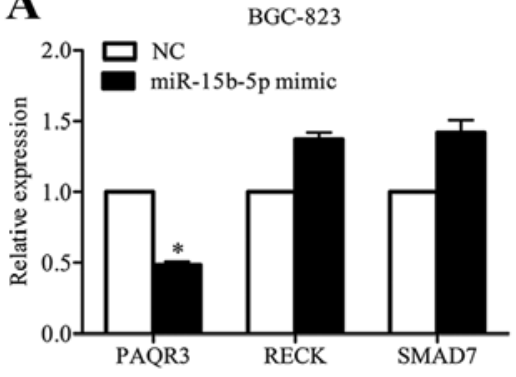

SGC-7901

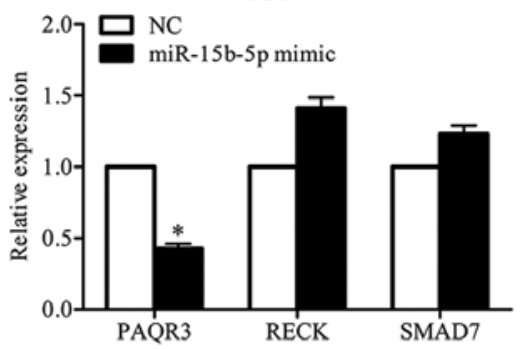

B

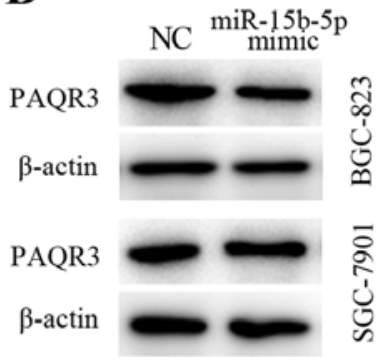

D

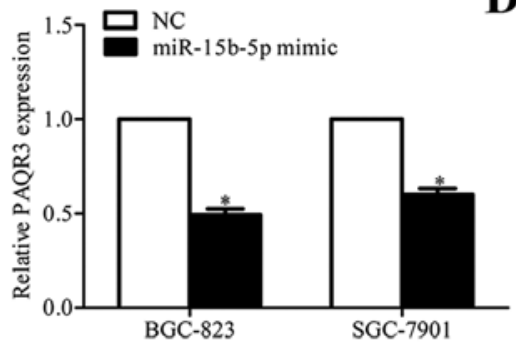

C
PAQR3-WT 5' UUAAAACUACUAAUUUGCUGCUU 3', | | | | | | miR-15b-5p 3'ACAUUUGGUACUACACGACGAU 5' PAQR3-Mut 5' UUAAAACUACUAAUACAGACGAU 3'

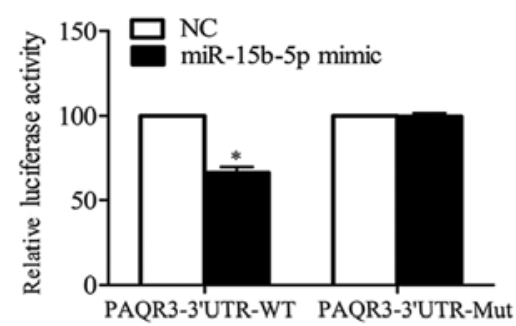

Figure 4. PAQR3 is a direct target of miR-15b-5p. (A) The mRNA level of PAQR3, RECK and SMAD7 in miR-15b-5p overexpressing GC cells. (B) The protein level of PAQR3 in miR-15b-5p-overexpressing GC cells. (C) The wild-type (WT) and mutant (Mut) binding site of miR-15b-5p on the 3'UTR of PAQR3. (D) The luciferase activity of cells which were transfected with pMIR-report-PAQR3-3'UTR-WT and miR-15b-5p mimics was much lower than that in the cells transfected with pMIR-report-PAQR3-3'UTR-Mut and miR-15b-5p mimics; ${ }^{\mathrm{P}}<0.05$ vs. NC. NC, negative control. 
A

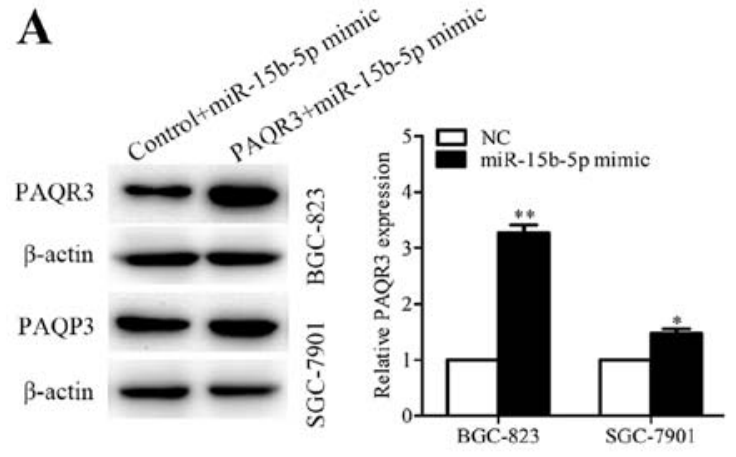

B

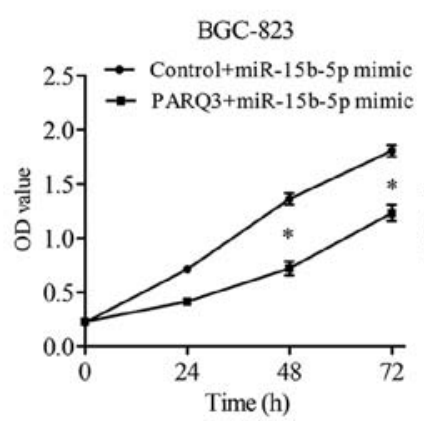

SGC-7901

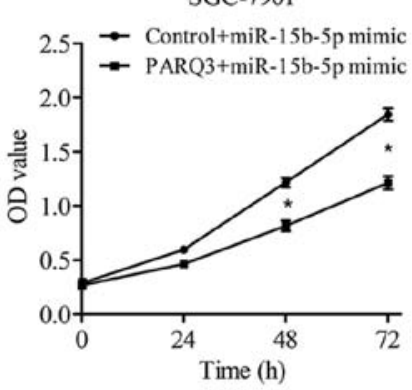

C

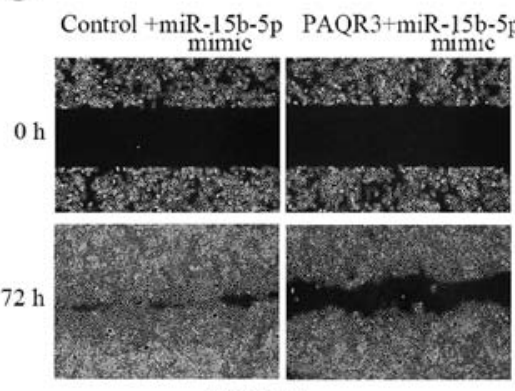

BGC- 823

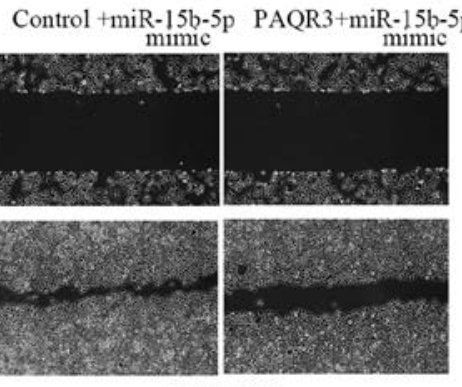

D

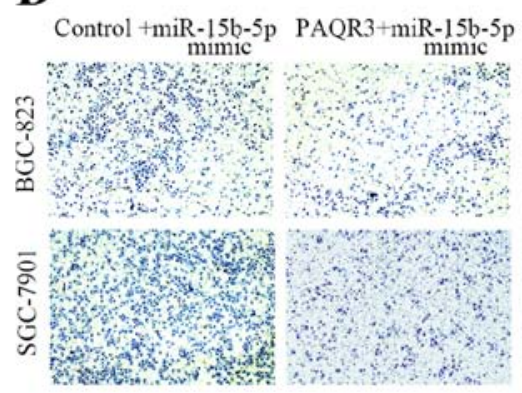

SGC-7901
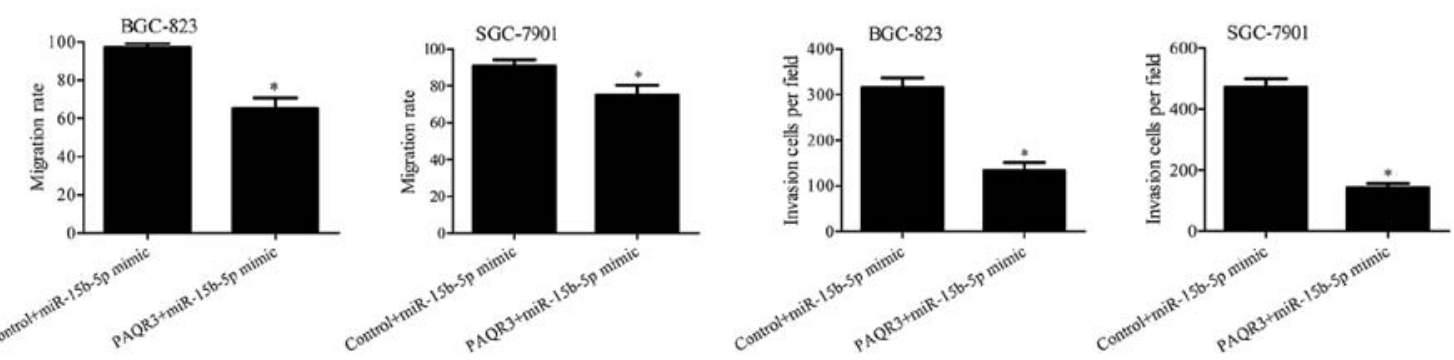

Figure 5. Overexpression of PAQR3 partially reverses miR-15b-5p-induced cell proliferation, migration and invasion. (A) The protein level of PAQR3 after transfection of the PAQR3 vector in the miR-15b-5p-overexpressing GC cells. (B) CCK-8 assay indicating the effect of PAQR3 on the proliferation of miR-15b-5p-overexpressing GC cells. The effects of PAQR3 on cell migration and invasion were analyzed by (C) wound healing and (D) Transwell assays in the miR-15b-5p-overexpressing cells; ${ }^{*} \mathrm{P}<0.05,{ }^{* *} \mathrm{P}<0.01$.

miR-15b-5p-overexpressing BGC-823 and SGC-7901 cells, and the efficiency was detected by western blotting (Fig. 5A). In addition, CCK-8, wound healing and Transwell assays were performed, and the results showed that overexpression of PAQR3 partially abrogated the enhanced cell proliferation (Fig. 5B), migration (Fig. 5C) and invasion (Fig. 5D) induced by miR-15b-5p overexpression.

\section{Discussion}

Previous studies have shown that miR-15b modulates multidrug resistance (15) and induces apoptosis (16) in gastric cancer (GC). However, studies concerning miR-15b-5p in GC metastasis are limited.

In the present study, the microarray analysis in GC tissues displayed numerous abnormally expressed miRNAs. Among these, miR-15b-5p was chosen for further research. The qRT-PCR results showed that miR-15b-5p was markedly increased in both GC cell lines and tissues. Moreover, high miR-15b-5p expression was found to be correlated with lymph node metastasis and degree of tumor invasion.
Recent studies have revealed that miRNAs can be potential markers of cancer for their stability in tissues and blood samples $(17,18)$. Chen et al found that circulating miR-15b in serum could be a potential marker for detecting hepatocellular carcinoma (19). Given that miR-15b-5p was correlated with lymph node metastasis and degree of tumor invasion, we aimed to ascertain whether the plasma miR-15b-5p level could be a biomarker of GC metastasis. The results showed that the plasma levels of miR-15b-5p in GC patients were higher than those in the healthy control, and patients with distant metastasis showed a higher miR-15b-5p plasma level than the non-metastasis patients. The results implied that high plasma miR-15b-5p may serve as a metastatic marker of GC. Due to the small size of the GC plasma samples, the results should be validated in a larger sample size.

In GC cell lines, the effects of miR-15b-5p on GC metastasis were studied by performing gain-of-function assays. We found that miR-15b-5p overexpression enhanced cell proliferation, migration and invasion. EMT is a critical process in tumor metastasis $(20,21)$. Thus, we assessed whether miR$15 b-5 p$ could facilitate EMT. The expression of EMT markers 
was detected and the results revealed that upregulation of miR-15b-5p promoted the EMT process. These results indicated that miR-15b-5p participates in GC metastasis partly by promoting cell proliferation, migration, invasion and EMT.

Since miRNAs exert their function by downregulating target genes (22), the targets of miR-15b-5p in GC cells were investigated. Three metastasis-associated genes (PAQR3, RECK and SMAD7) were identified as potential miR-15b-5p targets by online software programs and the expression levels of those selected genes were validated in miR-15b-5poverexpressing GC cells. Overexpression of miR-15b-5p in GC cells inhibited the expression of PAQR3, but not RECK and SMAD7. Luciferase assay indicated that PAQR3 is a direct target of miR-15b-5p. Furthermore, we found that overexpression of PAQR3 partially rescued miR-15b-5pinduced cell proliferation, migration and invasion. PAQR3 is a tumor-suppressor gene (23) that suppresses the proliferation, migration, tumorigenicity, EMT and metastasis in different types of cancers (24-27). In the present study, we found that miR-15b-5p promoted GC cell metastasis by targeting PAQR3.

In summary, the present study demonstrated that miR$15 b-5 p$ is upregulated in GC cell lines, GC patient tissues and plasma. A high plasma miR-15b-5p level may act as a biomarker for GC metastasis. miR-15b-5p overexpression enhances GC metastasis by regulating $\mathrm{PAQR} 3$ expression.

\section{Acknowledgements}

The present study was supported by the Science and Technology Development Plan of Jiangning District (grant no. 2014Dk04), and the Nanjing Health Bureau Research Project (grant no. YKK14194).

\section{References}

1. Chen W, Zheng R, Baade PD, Zhang S, Zeng H, Bray F, Jemal A Yu XQ and He J: Cancer statistics in China, 2015. CA Cancer J Clin 66: 115-132, 2016.

2. Torre LA, Bray F, Siegel RL, Ferlay J, Lortet-Tieulent J and Jemal A: Global cancer statistics, 2012. CA Cancer J Clin 65: 87-108, 2015.

3. Wang H, Zhang H, Deng P, Liu C, Li D, Jie H, Zhang H, Zhou Z and Zhao YL: Tissue metabolic profiling of human gastric cancer assessed by ${ }^{1} \mathrm{H}$ NMR. BMC Cancer 16: 371, 2016.

4. Jung J, Jung Y, Bang EJ, Cho SI, Jang YJ, Kwak JM, Ryu DH, Park S and Hwang GS: Noninvasive diagnosis and evaluation of curative surgery for gastric cancer using NMR-based metabolomic profiling. Ann Surg Oncol 21 (Suppl 4): S736-S742, 2014

5. Zhao X, Wang Y, Deng R, Zhang H, Dou J, Yuan H, Hou G, Du Y, Chen Q and $\mathrm{Yu}$ J: miR186 suppresses prostate cancer progression by targeting Twist1. Oncotarget 7: 33136-33151, 2016.

6. Farazi TA, Hoell JI, Morozov P and Tuschl T: MicroRNAs in human cancer. Adv Exp Med Biol 774: 1-20, 2013.

7. Shen ZL, Wang B, Jiang KW, Ye CX, Cheng C, Yan YC, Zhang JZ, Yang Y, Gao ZD, Ye YJ, et al: Downregulation of miR-199b is associated with distant metastasis in colorectal cancer via activation of SIRT1 and inhibition of CREB/KISS1 signaling. Oncotarget 7: 35092-35105, 2016.
8. MacLean JA II, King ML, Okuda H and Hayashi K: WNT7A regulation by miR-15b in ovarian cancer. PLoS One 11: e0156109, 2016.

9. Li J, Chen Y, Guo X, Zhou L, Jia Z, Tang Y, Lin L, Liu W and Ren C: Inhibition of miR-15b decreases cell migration and metastasis in colorectal cancer. Tumour Biol 37: 8765-8773, 2016.

10. Zhang Y, Huang F, Wang J, Peng L and Luo H: MiR-15b mediates liver cancer cells proliferation through targeting BCL-2. Int J Clin Exp Pathol 8: 15677-15683, 2015.

11. Sun G, Yan S, Shi L, Wan Z, Jiang N, Li M and Guo J: Decreased expression of miR-15b in human gliomas is associated with poor prognosis. Cancer Biother Radiopharm 30: 169-173, 2015.

12. Zhang WL, Zhang JH, Wu XZ, Yan T and Lv W: miR-15b promotes epithelial-mesenchymal transition by inhibiting SMURF2 in pancreatic cancer. Int J Oncol 47: 1043-1053, 2015.

13. Zhou RP, Chen G, Shen ZL and Pan LQ: Cinobufacin suppresses cell proliferation via miR-494 in BGC-823 gastric cancer cells. Asian Pac J Cancer Prev 15: 1241-1245, 2014

14. Livak KJ and Schmittgen TD: Analysis of relative gene expression data using real-time quantitative PCR and the $2^{-\Delta \Delta C_{\mathrm{T}}}$ method. Methods 25: 402-408, 2001.

15. Xia L, Zhang D, Du R, Pan Y, Zhao L, Sun S, Hong L, Liu J and Fan D: miR-15b and miR-16 modulate multidrug resistance by targeting BCL2 in human gastric cancer cells. Int J Cancer 123: 372-379, 2008

16. Sun H, Meng X, Han J, Zhang Z, Wang B, Bai X and Zhang X: Anti-cancer activity of DHA on gastric cancer - an in vitro and in vivo study. Tumour Biol 34: 3791-3800, 2013.

17. Mitchell PS, Parkin RK, Kroh EM, Fritz BR, Wyman SK, Pogosova-Agadjanyan EL, Peterson A, Noteboom J, O'Briant KC, Allen A, et al: Circulating microRNAs as stable blood-based markers for cancer detection. Proc Natl Acad Sci USA 105: 10513-10518, 2008.

18. Jiang X, Du L, Wang L, Li J, Liu Y, Zheng G, Qu A, Zhang X, Pan $\mathrm{H}$, Yang Y, et al: Serum microRNA expression signatures identified from genome-wide microRNA profiling serve as novel noninvasive biomarkers for diagnosis and recurrence of bladder cancer. Int J Cancer 136: 854-862, 2015.

19. Chen Y, Chen J, Liu Y, Li S and Huang P: Plasma miR-15b-5p, miR-338-5p, and miR-764 as biomarkers for hepatocellular carcinoma. Med Sci Monit 21: 1864-1871, 2015.

20. Dai J, Qian C, Su M, Chen M and Chen J: Gastrokine-2 suppresses epithelial mesenchymal transition through PI3K/AKT/GSK3beta signaling in gastric cancer. Tumour Biol 37: 12403-12410, 2016.

21. Zhang Y, Du J, Zheng J, Liu J, Xu R, Shen T, Zhu Y, Chang J, Wang $\mathrm{H}$, Zhang Z, et al: EGF-reduced Wnt5a transcription induces epithelial-mesenchymal transition via Arf6-ERK signaling in gastric cancer cells. Oncotarget 6: 7244-7261, 2015.

22. Bartel DP: MicroRNAs: Genomics, biogenesis, mechanism, and function. Cell 116: 281-297, 2004.

23. Yu X, Li Z, Chan MT and Wu WK: PAQR3: A novel tumor suppressor gene. Am J Cancer Res 5: 2562-2568, 2015.

24. Ling ZQ, Guo W, Lu XX, Zhu X, Hong LL, Wang Z, Wang Z and Chen Y: A Golgi-specific protein PAQR3 is closely associated with the progression, metastasis and prognosis of human gastric cancers. Ann Oncol 25: 1363-1372, 2014.

25. Li Z, Ling ZQ, Guo W, Lu XX, Pan Y, Wang Z and Chen Y: PAQR 3 expression is downregulated in human breast cancers and correlated with HER2 expression. Oncotarget 6: 12357-12368, 2015.

26. Huang W, Guo W, You X, Pan Y, Dong Z, Jia G, Yang C and Chen Y: PAQR3 suppresses the proliferation, migration and tumorigenicity of human prostate cancer cells. Oncotarget: Jun 3, 2016 (Epub ahead of print). doi: 10.18632/oncotarget.9807.

27. Guo W, You X, Xu D, Zhang Y, Wang Z, Man K, Wang Z and Chen Y: PAQR3 enhances Twist1 degradation to suppress epithelial-mesenchymal transition and metastasis of gastric cancer cells. Carcinogenesis 37: 397-407, 2016. 